\title{
De la violence verbale, pour une sociolinguistique des discours et des interactions ${ }^{1}$
}

\author{
Nathalie Auger \\ Université Montpellier 3 \\ Béatrice Fracchiolla \\ Université Paris 8 \\ Claudine Moïse \\ Université d'Avignon \\ Christina Schultz-Romain \\ IUFM d'Aix-Marseille
}

\section{Introduction}

Depuis quelques années nous cherchons à cerner dans sa plus grande complexité la notion de violence verbale, largement utilisée dans les médias et dans le cadre de l'Education Nationale, quand se généralisent les discours sur l'insécurité, la violence et les difficultés d'intégration. Les actes de langage menaçants (Vincent, 2005) constituent un large champ investi par différentes équipes de recherche de Chambéry à Bruxelles ou Québec pour le monde francophone et participent de la violence verbale. On peut citer, entre autres, l'insulte, le mépris, la menace, le dénigrement, voire la médisance (Mougin, 2006). Si nous nous intéressons à tous ces phénomènes et avons travaillé sur le malentendu (Auger et Moïse, 2005 ; Auger, 2005) ou l'insulte par exemple, nous essayons aussi de définir un processus global qui se manifeste dans des "montées en tension », analysables d'un point de vue interactionnel et où se jouent rapports de pouvoir, processus de catégorisation et actions sociales.

Il s'agit ici de présenter une synthèse des approches théoriques et méthodologiques qui nous aident à appréhender notre objet de recherche qu'est la violence verbale. Alors que nous avons largement publié des analyses d'interactions en milieu scolaire, informel ou à partir d'extraits de films, que nous avons explicité également nos visées sociales ${ }^{2}$, nous nous centrerons ici sur notre démarche théorique. Même si nous allons ponctuer notre présentation de quelques données empiriques, le lecteur pourra se référer à nos nombreuses analyses d'interactions sur un terrain français, disponibles à travers nos articles rassemblés sur le site de l'université d'Avignon autour de la violence verbale.

\section{Principes méthodologique}

\subsection{Ancrage sociolinguistique}

Nous avons, à diverses reprises (Moïse, 2002, 2003, 2007), posé nos « intentions sociolinguistiques ». Les différents champs de la sociolinguistique relatifs à l'histoire même de la discipline sont divers, allant du variationnisme qui s'est emparé de l'étiquette «sociolinguistique » (Chambers, 1994), à celui plus mouvant d'une sociologie du langage. Dans un sens, il est une première sociolinguistique qui s'intéresse à la société pour ce qu'elle nous dit sur la langue. C'est prendre souvent les différences sociales à travers des catégories préétablies, essentialistes (sexe, âge, origine, catégorie socio-professionnelle) dans une forme de réduction nécessaire, maniable et pratique, et s'en servir pour lire les variations en langue. La sociolinguistique française, marquée par l'histoire de la discipline et donc par la linguistique 
structuraliste, s'est longtemps, par frilosité aussi, limitée à une dimension descriptive. L'autre sociolinguistique (ou les autres sociolinguistiques) qui nous intéressent davantage disent la société à travers l'étude de la langue, des pratiques et des discours (Heller, 2002). Serait-ce une sociologie du langage, terminologie très (trop) associée à J. Fishman dès les années 60, critiquée d'ailleurs aujourd'hui (Williams, 1992) ou une anthropologie linguistique ? Mais cette autre sociolinguistique englobe alors un champ vaste, plus significatif dans le monde anglo-saxon (Mesthrie, 2001), allant de l'analyse des discours en œuvre dans la société à l'analyse des interactions notamment, voire à une nouvelle façon d'aborder la variation comme ressource en contexte (Gadet, 2000). C'est, de façon globale, cette sociolinguistique qui constitue notre cadre de recherche.

\subsection{Méthodologie de l'enquête}

Nous considérons le terrain, au-delà du site, et avec mes collègues sociolinguistes, de Didier de Robillard à Cyril Trimaille, comme des matériaux empiriques et des réseaux de relation à jamais circonscrits, ou alors délimités pour les besoins humains de l'enquête. Dans une telle optique, on ne pourra être qu'en exploration, à jamais insatisfaits, en prise avec une réalité que nous participons à construire. Nous avons mené une première enquête dans un collège de Zone d'Education Prioritaire à Perpignan dans le sud de la France. L'étude portait sur la violence verbale dans le cadre institutionnel et scolaire et il s'agissait d'analyser ses effets dans des rapports asymétriques. Selon une démarche caractéristique en ethnographie de la communication, l'enquêteur est en position d'observateur, participant mais distant toutefois du groupe à observer n'en détenant pas lui-même les codes. Il utilise prises de notes, consultation de tout document notamment ceux produits par les institutions, enregistrements de moments de tensions, de conversations authentiques, spontanées mais aussi d'interviews semi-dirigées, rendant compte des jugements, représentations, explications des locuteurs sur leur propre production verbale; il reste conscient de l'importance des interactions sociales et langagières, productrices de sens. Dans une perspective ethnométhodologique, la construction sociale n'est pas une donnée stable et préexistante, indépendante des interactions sociales, mais se modèle et module au gré des actions humaines, des savoirs, des apprentissages et des croyances de chacun. En examinant les comportements de l'intérieur, en faisant une observation attentive des processus par lesquels les acteurs interprètent constamment la réalité et réinventent la vie, on peut dresser des configurations sociales et une production singulière des interactions et, dans notre cas, de la violence verbale. Par la suite les terrains que nous avons investis, enquêtes en milieu informel (conversations familières, interactions dans les transports en commun, etc.) et extraits de scénarios de films, nous ont permis d'affiner nos analyses et de saisir la force du contexte interactionnel.

\subsection{La subjectivité du chercheur}

Autour d'un tel objet, la violence verbale, le chercheur lui-même, dans une démarche empirico-inductive, est partie prenante des situations d'interactions et sa propre subjectivité participe à la fois de la configuration du réel et de l'analyse (Moïse, 2007a). Ce terrain, objet d'engagement, pointe inéluctablement la grande part de subjectivité qui nous anime, qui nous fait acteurs de notre recherche, participant des interactions, orientant et situant la parole en circulation, au-delà de nous et contre nous parfois, composante dont on ne peut s'abstraire dans l'analyse (Auger et Moïse, 2005). L'un des objectifs de notre recherche est de prendre en compte la subjectivité comme une composante essentielle de l'analyse. P. Blanchet (2000 : 90-91) rappelle «[...] la nécessité d'une implication consciente du sujet chercheur [...] qui ne saurait être utilitariste »; Monica Heller (2002:10) complète le propos en proposant que «[...] le positionnement du/de la chercheur(e) fasse partie de la réflexion. La recherche doit donc comprendre sa propre action et son propre positionnement vis-à-vis de la question posée et des gens concernés, c'est-à-dire qu'elle doit adopter un point de vue critique face à elle-même. »

Dans les situations de tensions et de violence verbale, le chercheur pourra prendre position selon le contexte d'énonciation, rôle de témoin, de médiateur, d'observateur, de provocateur, d'acteur de la violence verbale. Si le chercheur n'est pas partie prenante, par sa présence même et notamment en 
milieux formel et scolaire, il lui est demandé souvent d'être témoin voire médiateur. Un des procédés fondamentaux mis en œuvre par l'école de Palo Alto (Watzlawick, Weakland, et Fisch. 1975), en particulier, celui qui consiste à régler un problème en sortant du cadre de la pensée auquel nous sommes généralement habitués : pour rompre certains schémas de communication, il faut ainsi s'extraire de la binarité du rapport, prendre de la distance et aller, d'un point de vue géométrique, chercher la tierce solution à l'extérieur. Plus simplement, il s'agit d'insérer un point de vue tiers dans une relation binaire. L'idée d'une psychosociolinguistique (Van Hooland, 2002) requiert la présence d'un tiers dans la communication - la partie «psycho » - qui joue un rôle mixte de réflecteur, mitigeur, mise à distance et objectivation par comparaison; qui interroge du moins ce qui est donné pour réel par le locuteur à l'interlocuteur. Si l'on comprend la notion de sociolinguistique relativement à la dimension sociale, spatiale et temporelle, on peut comprendre alors la dimension «psycho » dans le sens d'une nécessaire présence médiatrice (physiquement présente ou non) entre les deux individus qui élaborent un type d'échange fonctionnant jusque-là en cercle binaire et fermé. Cette idée est intéressante dans la mesure où elle réintroduit l'idée d'un triangle correspondant à la réalité pronominale et grammaticale (je, tu, il/elle ) en redonnant une véritable place de personne au «il/elle», alors que pour les interactions verbales, on s'attache(ait?) essentiellement à la relation binaire, entre « je» et « tu » en traitant le « il»/ « elle» comme une «non-personne ». Il est probable qu'une telle perspective annonce un changement à l'œuvre en ce qui concerne les représentations de l'altérité à travers de nouvelles formes d'interactions. On peut remarquer à cet égard l'importance donné ces dernières années au rôle (nouveau) du médiateur, du négociateur, qui apparaît dans la résolution non violente des conflits (Auger, Fracchiolla, Moïse 2005).

En milieu informel, le chercheur peut être participant d'une interaction violente voire peut la provoquer à des fins d'expérience. Mais, du point de vue de la matérialité de l'objet, il est souvent difficile de saisir promptement son matériel d'enregistrement. Le mieux, surtout si l'on est soi-même partie prenante de la violence verbale, qu'on veuille ou non entrer dans le jeu interactionnel, est de faire confiance à sa mémoire pour tenter de retranscrire au mieux par la suite les interactions (Moïse, 2007b). Parfois, il est possible d'enregistrer, mais il manque souvent la première phase de montée en tension. Il s'agit aussi de contrôler ses émotions et sa propre violence intérieure. Un tel projet, qui permet aussi de mener des interventions de formation dans un cadre de recherche action, demande une capacité réflexive du chercheur face à ses comportements et pratiques. Dans une telle perspective, notre équipe de recherche a dû se former à la «gestion de conflits » et à la «communication non violente », reposant, au-delà de l'analyse et de la compréhension des phénomènes langagiers, sur une appréhension psychologique des relations verbales.

\section{Méthodologie de l'analyse}

Nous ne pourrons ici développer tous les outils ni leurs ancrages épistémologiques que nous utilisons pour décrire la violence verbale. Mais, au fil des recherches et analyses, et dans une perspective globale et intégrative, il nous est apparu nécessaire, pour une meilleure compréhension de notre objet, de faire appel à plusieurs approches théoriques, entre analyse de discours et analyse conversationnelle.

\subsection{D'une perspective sémiotique à l'analyse des discours}

\subsubsection{Discours multimodal et sphère sémiotique}

De notre point de vue, la violence verbale, comme toutes formes d'interactions sociales et langagières, n'est pas à considérer seulement en langue mais aussi dans une dimension discursive et spatiale (Budach, Moïse, Duchêne et Richards, 2007). Elles rendent compte, dans une perspective de sémiotique sociale, d'une «construction de sens » (meaning making) (Halliday, 1978) à lire et à saisir dans sa globalité. Partie prenante dans une construction multimodale (visuelle, verbale, graphique, architecturale) des interactions et des changements en jeu dans l'espace urbain, ils sont alors à juger dans leur dynamique, leur temporalité éphémère, emblèmes d'un paysage sémiotique (semiotic landscape) (Kress et van 
Leeuwen, 1996), signifiants de l'événement. L'événement (Hymes, 1974 ; Heller, 1995) renvoie à un moment d'interaction sociale précis qui s'inscrit dans un contexte spatial et temporel déterminé et qui réunit un ensemble d'acteurs spécifiques. L'événement représente un moment particulier dans une série d'actions du même type; il est construit sur l'expérience et reconnaissable comme tel par les membres d'une communauté partageant les mêmes valeurs et pratiques culturelles. Les interactions et les interactants sont donc pris dans une multitude de liens et de relations qui participent de l'élaboration interactionnelle, au-delà des routines et des contraintes conversationnelles. Ainsi, l'interaction sociale, qui se déroule dans un cadre événementiel particulier, repose sur un nombre de pratiques sociales ritualisées qui rendent compte des intérêts des acteurs et de leurs expériences, en écho avec d'autres interactions antérieures du même type. Ainsi, nous avons vu que chacun moment de violence verbale, donc chaque montée en tension, s'incrivait dans un cadre singulier avec des acteurs investis de leur rôle, et déterminant les actes violents. La violence entre enseignants et élèves se joue à des moments institutionnels et événementiels particuliers de conflit; les slogans contre les hommes politiques (Corpus Fracchiola, Moïse, 2006), « Chirac, patron des enfoirés », « Jeu de mains, jeu de Villepin », ont pris leur force et leur sens lors des dernières manifestations d'avril 2006 contre le Contrat Première Embauche (Fracchiolla et Moïse, à paraître).

\subsubsection{Contexte}

Les analyses interactionnelles posent de façon traditionnelle le contexte extra-discursif et le contexte intra-discursif. L'ethnométhodologie pose comme un de ses principes (outre la réflexivité - capacité des locuteurs à interpréter les signes qu'ils observent pour construire du sens et donc les interactions - et l'accountability - les faits sont reconnaissables et donc analysables comme tels), celui de l'indexicalité, en référence au contexte interlocutif, et s'attache donc essentiellement à l'analyse située des interactions. Dans cette perspective, le contexte préexiste moins à l'interaction que l'interaction ne le détermine et dévoile les éléments pertinents à considérer dans l'analyse, puisque donnés par les locuteurs eux-mêmes. C'est à travers l'analyse des tours de paroles et des indices observables, et non des informations extérieures, que l'on peut saisir comment les locuteurs usent du contexte et donc construisent du sens. Pour notre part, si nous ne nions pas que le sens se construit dans l'interaction, nous considérons que le contexte et donc le positionnement des acteurs nous permettent de mieux comprendre d'une part les mécanismes interactionnels (l'élaboration des thèmes ou topics, les réparations, les évitements, renoncements ou collaborations) constitutifs d'un certain déroulement de la violence verbale et d'autre part les enjeux sociaux de la violence verbale. Ainsi, la violence verbale est signifiée d'un côté par le contexte d'énonciation (le co-texte), mais d'un autre côté par le contexte social, ethnique, médiatique, symbolique voire psychologique. Ainsi, le contexte interactionnel est très vaste, allant du contexte immédiat (où se déroule une altercation) aux discours politiques en circulation par exemple, et dans une polyphonie certaine. Il existe donc des contextes hiérarchisés et même si, en leur temps, Fishman et Hymes en ont proposé des taxinomies, il reste toujours, il est vrai, difficile d'en délimiter les frontières (Heller, 1995) et d'en cerner parfois la réelle pertinence. Les interactions et les interactants sont pris dans une multitude de liens, de relations particulieres qui participent de l'élaboration interactionnelle, au-delà des routines et des contraintes conversationnelles (Moïse, 2007b). Ainsi, dans les altercations dans la rue, sous couvert d'anonymat et face au regard public, la violence est souvent fulgurante et les thématisations autour d'arguments objectivés sont réfutées pour basuler dans des atteintes à l'identité, comme le montre une altercation entre une automobiste et un piéton (Corpus Moïse 2006) au sujet d'un papeir jeté par terre. Après avoir balayé les arguments sur la propreté ou la citoyenneté, la conductrice lance un " tu es vilaine », puis suite au «ne me tutoyez pas », un « occupe-toi de ta vie et de ton cul / je t'emmerde connasse » qui clôt par une insulte la séquence interactionnelle.

\subsubsection{Circulation des discours et polyphonie}

Si le champ du discours rapporté est complexe (Authier-Revuz, 1992 ; Rosier, 1999), il s'agit dans le cadre de la violence verbale de voir ce qui fait «circulation » (Fracchiolla, Moïse, à paraître), et cela audelà d'une approche "pragmatique ou matérialiste posant la nécessaire primauté d'un discours à citer, 
c'est-à-dire qu'il y aurait nécessité pour avoir du discours rapporté d'un discours antérieur que l'on cite, que l'on résume, reformule voire trahit » (Rosier, 2005 : 157). En même temps, et pour avoir largement travaillé en ce sens (Heller, 2002 ; Fairclough, 1992) nous entendons par "circulation des discours » la part constitutive pour un discours donné des objets et idéologies antérieures, idéologies portées par les discours hégémoniques. Au sens où pour Michel Foucault un discours est «constitué par l'ensemble de tous les énoncés effectifs dans leur dispersion d'événements et dans l'instance qui est propre à chacun » (1994, I : 705). Qu'est-ce qui se voit alors reproduit dans les actes et arguments violents ? Qu'est-ce qui est de l'ordre des pensées dominantes en œuvre, prises dans leur historicité ? Qu'est-ce qui peut alors faire violence ? Comment circulent insultes et menaces? Ainsi, nous avons analysé le phénomène de la circulation des discours à travers les manifestations sur le CPE (Fracchiolla et Moïse, à paraître), et celui plus précisément du dialogisme à travers le traitement de la question de la violence scolaire dans la presse nationale et régionale (Auger, 2007). Si nous reprenons la citation de M. Bakhtine (1977 : 106) à propos du dialogisme, celui-ci implique que «toute énonciation, même sous sa forme écrite figée, est une réponse à quelque chose et est construite comme telle [...]. » En effet, tout discours renvoie à des discours précédents, soit de manière implicite (thématique par exemple), soit de manière explicite grâce à des marques linguistiques qui nous permettent de reconstruire cet/ces autre(s) discours (discours rapporté, citations etc. ou en polyphonie plus discrète parfois). Dans la manifestation d'avril 2006 (Fracchiolla, Moïse, à paraître) Une pancarte intitulée « Les vrais casseurs », montrant trois photos respectivement de N. Sarkozy (sur la gauche) « Karsherator», de J. Chirac au milieu « Super menteur », et de D. de Villepin «Villepintator». On peut noter l'utilisation de plusieurs références en faisceau de sens : les renvois récurrents à une filmographie de science-fiction actuelle opposant le bien et le mal (Superman, Terminator, La guerre des Etoiles). Ces références permettent de figurer de manière spécifique l'affrontement entre les forces du bien et du mal : les hommes politiques sont représentés, sous forme de marionnettes, comme autant d'anti-héros, vieillissant, ambitieux, et ayant «basculé » du côté du mal, avec une identification déclarée du pouvoir à la figure de «l'Empereur» et de Darkvador, dans l'ensemble de la saga de La guerre des étoiles; et, par jeu de miroir, l'association des « jeunes » et des manifestants aux bons « Jedi », redresseurs de torts et garants du Bien dans l'Univers. Cette stratification synchronique des discours circulant s'élabore également à travers les néologismes de noms « Karsherator » (Sarkozy) et «Villepintator» (Villepin), lesquels néologismes jouent également sur les mots. Karsher est ici un terme repris en focalisation d'une phrase prononcée par Nicolas Sarkozy pendant des violences urbaines et lors de sa visite dans une cité d'Argenteuil, quelques mois auparavant, le 5 novembre 2005 : " Vous avez assez de cette bande de racailles ? Eh bien, on va vous en débarrasser ». C'était alors après sa promesse de la Courneuve le 20 juin 2005 de «nettoyer au Kärcher la cité des $4000 »$, expression violente, ressentie comme telle, et reprise comme telle que l'on retrouve encore sur une pancarte à travers le mot valise autour de Sarkozy, racaille et kärcher : « Nettoyons la France de la Sarkaïlle ». Ainsi, si N. Sarkozy est relégué en une $3^{\text {ème }}$ personne, se trouvant en effet ici, délocuté et donc tenu pour peu, (Karsherator=Karsher a tort), D. Villepin est lui en revanche apostrophé directement (Villepintator=Villepin t'as tort), dans une forme d'injonction, inversant les rapports de pouvoir.

Le dialogisme a également été étudié aussi au travers de discours constitués d'une part de la parole des principaux acteurs (enseignants, élèves, acteurs sociaux) sur la situation parfois conflictuelle qu'ils vivent quotidiennement, et d'autre part de discours en «acte» en classe (Auger, 2003). On obtient donc un « croisement» des paroles des uns et des autres qui permet de bien distinguer les représentations de la situation (sous la forme d'entretiens avec les différents acteurs) et les interactions effectives qui se produisent et qu'analyse le chercheur. Quand on étudie ces différents discours, on remarque que la situation est souvent vécue comme antagonique par les locuteurs et que cet antagonisme est fréquemment attribué en général à l'autre, celui qui n'est pas du groupe d'appartenance (l'élève s'il s'agit de l'enseignant, l'enseignant s'il s'agit de l'élève, et ainsi de suite). Le cadre d'analyse sera donc celui de l'AD (Analyse de Discours) et de l'AC (Analyse de Conversation), dans le sens où ces outils peuvent permettrent l'observation du lieu d'émergence de l'interdiscours. Ces études en contexte appuient donc les propos de Bakhtine sur le rôle du « mot» comme porteur des « accents sociaux à orientation contradictoire ». Dans nos corpus (Moïse, Auger 2001), on peut signaler une forme énonciative récurrente, dialogique : la métaphorisation animale ou surnaturelle qui vise à nier l'autre en lui refusant les attributs propre à 
l'humain (qui est une forme caractéristique de la stigmatisation dans les travaux de Françoise Héritier, 1999). Et si les difficultés du métier d'enseignant sont vécues comme un combat, l'élève en devient le protagoniste animal dans ses caractéristiques agressives et brutales : « On n'a pas fait ce métier pour être en combat dans une arêne hein » (Entretien d'enseignante, corpus Moïse, Auger 2001).

\subsection{De l'analyse conversationnelle}

\subsubsection{Le contrat communicationnel}

Le contrat communicationnel est ce qui permet aux partenaires d'un échange langagier de se reconnaître l'un l'autre avec les traits identitaires qui les définissent en tant que sujet de cet acte, de reconnaître la visée de l'acte qui les surdétermine de s'entendre sur ce qui constitue l'objet thématique de l'échange et de considérer la pertinence des contraintes matérielles qui déterminent cet acte (Charaudeau, P. et Maingueneau, D. 2002 : 138). Il est pour nous un élément important dans l'analyse de la violence verbale. Quand les indices de contextualisation (Gumperz, J, 1982/1989) ne sont pas respectés ni reconnus (ce qui doit être considéré comme interactions autorisées en salle de classe, comme formes d'adresse à un guichet de poste, dans un bus etc...), quand le contrat de communication est remis en question et malmené par l'un des locuteurs, il peut y avoir émergence de conflit. En situation de relation de pouvoir, l'impasse de la coopération et les marques d'affrontement montrent que les locuteurs, à travers l'usage qu'ils font de leur parole, renégocient sans cesse la place sociale qui leur est assignée. Dans des situations de dissymétrie (milieu scolaire par exemple) les tentatives avortées de négociation peuvent être analysées à deux niveaux différents, celui même des interactions, dans l'absence de relance, par exemple, de la parole de l'autre (quand pendant de nombreuses interactions une enseignante ne répond pas à un élève qui l'interpelle « madame, madame » - corpus Moïse, Auger, 2001), dans le non respect des prises de parole, mais également au niveau des télescopage de normes plus sociales (un élève qui est dans une demande de « conversation » alors que l'enseignante reste dans un modèle hiérarchique - corpus Moïse, Auger, 2001) que strictement linguistiques. De cette façon, on doit prendre en compte, au-delà des jeux menés dans l'espace interactionnel (gestuelle, intonation, enchaînement, etc), ce qui dépend de l'intersubjectivité des locuteurs, façonnée par des expériences psychologiques et sociales singulières, des parcours de vie, et qui se rejouent sans cesse dans l'interaction dans des compromis, malentendus, négociations, dans des jeux d'affrontements, d'image de soi et d'influences réciproques.

\subsubsection{Les représentations sociales}

Il est intéressant de comprendre alors comment la montée en tension est liée aussi (comme toute interaction d'ailleurs) à la représentation que l'on se fait de l'autre, (je parle en fonction de ce que je me représente de l'autre et de ce que je me représente de ce que l'autre se représente de moi). Cette représentation pose l'autre dans des formes stéréotypées et réductrices, formes qui se rejouent et se reconstruisent sans cesse dans l'interaction (puisque tu es comme ça, je ne peux te parler autrement). Les représentations sont à la fois sociales et interculturelles et reposent inéluctablement sur la représentation identitaire de l'autre opposée au même (Fracchiolla, 2003). Ces représentations sont d'autant plus efficaces qu'elles sont réactivées, reprises par toute une production collective, qu'elles se diffusent au sein du groupe et le construisent dans des processus interactifs de catégorisation (ce qui « fait » élève, citoyen, client, etc.) (Mondada, 1998) et dans les discours rapportés en circulation. Comme le note (corpus Moïse, Auger, 2001) un surveillant au sujet des certains élèves jugés violents : " i(l)s ont des problèmes chez eux et $\mathrm{i}(\mathrm{ls})$ viennent à l'école pour avoir de nouveau d'autres problèmes et toi: mettons tu l'enfermes dans son carcan dans son truc quoi tu le traites déjà quan(d) il arrive de bandit: / ou de trucs comme ça les troisquarts n'auront même pas envie de prouver: qu'ils ne sont pas comme ça donc i(ls) vont jouer ce jeulà : //».

La violence verbale pourra émerger dans des refus de catégorisation et dans des renégociations du sens attribué. Ainsi, on peut dire que les interactions construisent, à un niveau social plus large, des espaces discursifs idéologiques (Heller, 2002) qui servent la reproduction de l'ordre établi et les pouvoirs 
légitimés. On sait alors que les interactions sont prises dans des formes d'interdiscursivité et dans des discours qui se répondent. Et pour suivre la citation devenue célèbre de M. Pêcheux $(1975: 147)$, le propre de toute formation discursive est de dissimuler, dans la transparence du sens qui s'y forme [...], le fait que «ça parle » toujours avant, ailleurs ou indépendamment. On peut alors reconnaître ce qui doit être des interactions entre un enseignant et un élève, entre un père et son fils (consensus idéologique construit par les discours en circulation autour de certaines notions comme la politesse, l'autorité etc.) et toute rupture des enchaînements interactionnels établis et attendus (non respect des tours de parole par exemple), si elle ne peut être renégociée dans l'interaction, pourra basculer dans la montée en tension. Dans la scène d'ouverture du film Karnaval (Auger, Moïse, 2005), suite à un mésentente culturelle, un fils va refuser l'attribution filiale pour rendre compte du sentiment de domination « un père ça / un père ça / un fils [il s'auto-désigne]/ moi je suis un esclave ici moi ».

\subsubsection{Les participants à l'interaction et les rapports de face}

Nous avons tenté de décrire l'interaction didactique en situation conflictuelle afin de proposer une description contextuelle de la violence verbale en milieu scolaire. La description de la gestion de la relation interpersonnelle à l'occasion de la remise en cause de la relation dissymétrique classique enseignante / élève, c'est-à-dire à l'occasion d'une tentative de déstabilisation voire d'un renversement du rapport de places classique enseignant / élève(s), a particulièrement attiré notre attention (Romain, 2006, 2007, 2008). Dans ce contexte, l'analyse porte sur les situations potentiellement menaçantes pour le « bon usage » de l'interaction didactique à partir du modèle de la politesse décrit par Brown et Levinson (1978, 1987) (considérations constitutives d'un modèle réaménagé par Kerbrat-Orecchioni, 1992), de la notion de face empruntée à Goffman (1973) et de l'analyse des places dans l'interaction conduite par Vion (1999) selon laquelle une relation interpersonnelle impliquant le langage repose sur cinq types de places (institutionnelles, modulaires, subjectives, discursives et énonciatives).

Les participants à l'interaction verbale ou plus précisément les rôles qu'ils sont tenus de jouer au moment du conflit déterminent les modalités des montées en tension. En situation hiérarchique de classe, la violence verbale est perçue comme une vive transgression des élèves par rapport aux normes scolaires Au-delà de l'impolitesse (et donc d'un manque de reconnaissance de l'autre) il s'agit de transgression et de rupture d'un ordre social, où les élèves usurpent leur statut d'élèves face à « l'autorité ». La violence verbale est dans ce cas beaucoup plus lourde de conséquences qu'en situation interpersonnelle et se voit susceptible de sanctions pour rétablir l'ordre menacé. Se rejoue alors à travers la violence verbale le rapport de forces symboliques en jeu entre les protagonistes où se manifestent les rapports de pouvoir et l'absence de normes partagées (Bourdieu, 1982). C'est que les protagonistes manifestent à travers leurs pratiques langagières et par les ressources qu'ils ont à leur disposition (en l'occurrence la violence verbale) les tensions sociales à l'œuvre. Selon la position sociale du locuteur dans l'interaction (enseignant, élève, passant, automobiliste), la violence verbale va se configurer différemment mais surtout rendre compte d'enjeux sociaux et de prises de risque différents.

Dans ce sens, nous nous appuyons sur l'analyse conversationnelle doublée également d'une approche lexicométrique, pour étudier certains corpus et notamment des corpus issus de situations de classe et des corpus politiques. Ces analyses se fondent d'une part sur une étude statistique des données textuelles réalisées à l'aide du logiciel Lexico $3^{3}$ et d'autre part sur une analyse énonciative et pragmatique fine, les deux types de résultats s'enrichissant et se complétant l'un l'autre. Les données statistiques permettent ici en particulier de faire des explorations objectives de type sémantique et structurel sur le texte et de faire ressortir certains éléments non toujours visibles à l'œil nu (Fracchiolla, 2003). L'analyse énonciative et pragmatique des actes de langage (Austin, 1962 ; Searle, 1972) permet parallèlement de révéler certaines constructions du sens conversationnel qui échappent totalement à une analyse statistique, fondée sur le compte des formes. Un corpus issu d'une centaine de rapports de professeur rédigés sur des élèves à partir de situations de violence verbale conversationnelle en classe est ainsi à l'étude. De même, des résultats particulièrement intéressants ont été obtenus pour l'analyse du débat Sarkozy-Royal en utilisant ce double 
processus d'analyse. Par l'usage surabondant de l'interpellation « Madame», Nicolas Sorkozy (115 occurrences pour 7 occurrences de « Monsieur » pour Ségolène Royal) joue de «l'attaque courtoise », en imposant sa domination polie (Fracchiolla, 2008).

\section{Les processsus de violence verbale}

Ainsi, notre approche qui repose sur plusieurs niveaux d'analyse nous a permis de définir un processus global de violence verbale, la «montée en tension ». On peut la définir comme un processus marqué par des «déclencheurs » et des «étapes séquentielles» spécifiques qui s'inscrit dans des actes de paroles repérables, des rapports de domination entre les locuteurs, des télescopages de normes et de rituels, des constructions identitaires et idéologiques.

\subsection{La montée en tension}

La violence verbale est inhérente au conflit qui est une divergence de points de vue, manifestes sur le plan interpersonnel et des normes sociales (il peut y avoir par exemple divergence sur la notion de « respect» liée aux nuisances sonores de voisinage) et qui entraîne une forte tension entre les locuteurs. La violence verbale est d'autant plus «radicale » qu'elle s'inscrit dans une opposition caractérisée entre les interlocuteurs. Nous avons donc dégagé, suite à différentes analyses de situations de violence verbale, les étapes suivantes constituant une montée en tension dans les conversations.

$1^{\text {ère }}$ étape : la violence potentielle. La violence verbale est à relier au contexte général de communication, forme de climat général (Galatalo, Mizzau, 1998). On parlera de violence potentielle, liée à la personne elle-même, à son agressivité comportementale, ou liée à un contexte supposé violent, construit à travers représentations ou mises en scènes médiatiques, comme le sont les images renvoyées des banlieues.

$2^{\text {ème }}$ étape : la violence embryonnaire ou amorce de la violence verbale. Comme nous l'avons montré par ailleurs, il est des éléments identifiables linguistiquement d'une amorce de la violence verbale. On peut noter parmi eux, l'agressivité avec changement prosodique et posture particulière du corps, le harcèlement verbal avec répétition interactionnelle dans différentes séquences conversationnelles, les joutes verbales caractérisées par des changements de registres verbaux. A ranger dans ces figures, toutes sujettes à l'intersubjectivité des locuteurs, l'impolitesse et l'incivilité. On considère l'impolitesse comme une rupture des rituels conversationnels interpersonnels (refus de dire bonjour) tandis que l'incivilité serait à prendre d'un point de vue des codes sociaux (utilisation du téléphone portable dans le train par exemple). L'amorce de la violence verbale est « lancée » par un locuteur A et va entraîner certains modes de réactions de la part du locuteur B.

$3^{\text {ème }}$ étape : la violence cristallisée. Face aux attaques de A, le locuteur B peut adopter différents comportements, notamment entrer résolument dans le conflit et prendre part à la montée en tension. Dans ce cas-là, il est fait usage de l'insulte, de la menace (souvent dans une forme d'injonction physique, «je vais te casser la tête »), du mépris, actes pragmatiques repérables dans le discours à forte valeur perlocutoire. La montée en tension se joue et se rejoue dans les différentes prises de parole des locuteurs sous formes de boucles interactionnelles où $\mathrm{A}$ et $\mathrm{B}$ interchangent leur place dans une joute verbale. Cette entrée marquée dans la violence verbale peut être dépassée ou évitée à travers d'autres résolutions conversationnelles. Le locuteur B peut tenter de mettre un terme au conflit par la négociation, qui portera sur l'objet même du conflit ou sur la relation interpersonnelle (Ott, 1998). Dans ce cas, il faut que les deux locuteurs soient capables de "s'entendre » hors de tout sentiment d'atteinte à la face. D'une autre façon, le locuteur B peut décider d'opter pour la fuite ou l'évitement. La fuite consiste à se taire, voire à physiquement partir, ou à opter pour un changement thématique ( «bon parlons d'autre chose »). L'évitement consiste à rester dans la thématique sans contre-attaquer, par exemple avec des marques d'humour. Véronique Traverso (1996) parle pour l'évitement de dispute évitée quand il y a désaccord 
sans négociation ni explicitation, chacun des locuteurs restant sur ses positions ; on est dans un échange immobile.

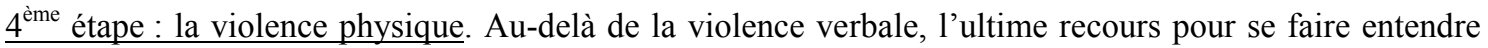
est la violence physique dans une forme de passage à l'acte souvent annoncé pragmatiquement - par la parole ou le mimo-gestuel - dans les montées en tension (« si tu continues, ça va mal se passer / je vais te casser la gueule »).

\subsection{Les déclencheurs de conflits}

Pour comprendre comment se met en place la violence verbale, c'est-à-dire une certaine montée en tension conversationnelle entre les locuteurs, il faut saisir ce que nous avons appelé les déclencheurs de conflits. Nous avons pu observer qu'ils étaient liés à différents aspects des conflits, liés aux relations interpersonnelles, structurelles ou culturelles. Le conflit de valeurs ou culturel (Ott, 1997). Les locuteurs sont en mésentente voire en opposition idéologique sur des représentations, des idées morales liées aux groupes sociaux ou ethniques. En ce sens, il va légitimer les différences et pourra s'orienter vers un conflit interpersonnel. Les différentes appréhensions des universaux culturels - relation à la famille, à la mort, au travail... - peuvent entraîner, dans des situations extrêmes d'incompréhension, des situations de conflit. Le conflit structurel s'actualise dans la transgression des normes sociales qui maintiennent l'ordre établi et qui sont donc particulièrement identifiables dans les structures institutionnelles, système scolaire, entreprises, etc. Dans ces situations, les contrats de parole (rituels conversationnels implicites liés à un contexte particulier) sont malmenés par la violence verbale, rupture provoquée et engendrée par des sentiments d'injustice et de relations dominants/dominés. Le conflit interpersonnel repose sur une remise en question de l'autre en «tu », dans un reproche de ce qu'il est, forme de conflit d'identité (Ott, 1997). On est dans le non-respect de la face, sans précaution locutoire et dans une recherche de l'avantage conversationnel pour un maintien ou prise d'une place haute.

\subsection{Les actes de langage}

Toute montée en tension se caractérise aussi par des actes de langage analysables d'un point de vue linguistique. En ce sens, nous nous sommes intéressés à des figures comme le malentendu, la menace, le harcèlement ou l'insulte (Moïse, à paraître). Nous voudrions ici exposer l'exemple de l'insulte, comme acte pragmatique de dernier recours à la violence verbale. L'insulte ne peut exister qu'en situation interactionnelle pragmatique, et non pas seulement en langue. "Merde », quelle que soit la forme de « transgression langagière », peut être à la fois mot grossier, juron et insulte, (Huston, 2002). Le gros mot jouera, en langue, sur la fonction référentielle du langage, fera référence donc à l'objet désigné («la merde $\gg)$. Le juron joue de la fonction expressive et sert le locuteur, pour ponctuer son discours, façon d'être dans l'emphase («merde ! »). La transgression sera d'ordre scatologique, sexuel (« Putain! ») ou sacré («Nom de Dieu! »). L’insulte seule vise l'interlocuteur dans une fonction impressive, «je te dis merde » ou même « tu es une merde », « espèce de merde ».

\subsubsection{Fonctionnement de l'insulte}

L'insulte apparaît le plus souvent en situation dissymétrique comme dernier recours avant la confrontation physique. Elle est en revanche plus fulgurante dans les situations informelles et symétriques et joue de façon plus directe avec la négation de l'autre. L'insulte est un acte de langage interlocutif; elle porte une force émotionnelle, voire pulsionnelle, et vit l'autre dans la volonté de le rabaisser et de le nier. Elle tient un rôle éminemment perlocutoire ( «Parce que je te traite de gros lard, tu vas te sentir comme ça »). Ce fonctionnement-là est rendu possible par des effets linguistiques. « Le trait axiologique est une propriété sémantique de certaines unités lexicales qui leur permet dans certaines circonstances de fonctionner pragmatiquement comme des insultes, le marqueur illocutoire (effet sur l'interlocuteur) de l'insulte étant la résultante complexe d'un ensemble de faits particuliers (Kerbrat-Orecchioni, 1997 : 79). » Parmi ces faits particuliers, il y a la valeur lexicale des lexèmes. Il semble évident que certains 
axiologiques négatifs puissent être réactivés pour faire insulte : " gros » sera plus activé que « mince » par exemple ${ }^{4}$. Il y a la forme syntaxique aussi, le terme péjoratif étant alors employé en fonction vocative avec notamment des modalités de catégorisation comme "espèce de ». Il y a aussi l'intonation, tout terme, même neutre, pouvant se charger d'insulte par la simple force d'évocation, façon de passer du constat à l'énoncé injurieux. En ce sens, l'insulte aura toujours une force illocutoire, puisqu'un effet doit être produit sur l'interlocuteur. Mais pour que l'insulte fonctionne pleinement, encore faut-il que l'interlocuteur la perçoive comme telle, en bref qu'elle touche, qu'elle déstabilise et non qu'elle conforte l'autre dans ses croyances. Comme le montre C. Kerbrat-Orecchioni (1997), si je traite l'autre d'anarchiste «Anarchiste!», et s'il répond «parfaitement», l'effet souhaité est rompu. Finalement, l'insulte est identifiable linguistiquement dans sa forme et vise l'autre dans un effet illocutoire voire perlocutoire dégradant et à travers une subjectivité partagée. Ainsi, les vannes se situent en marge des insultes, parce qu'elles reposent sur une connivence partagée, un jeu quasiment rhétorique, des rituels établis, des codes. Mais c'est un jeu à risque, et quand elles sont perçues comme blessantes, les vannes peuvent basculer dans l'insulte.

\subsubsection{L'insulte en situation dissymétrique}

Certaines formes d'insultes finalement n'en sont pas, comme le révèle l'étude des parlers dits jeunes. Ainsi, la forme «eh bâtard !» se trouve désémantisée, perd sa valeur illocutoire, pour servir de terme d'adresse, voire d'affection (Lagorgette et Larrivée, 2004) ou, parfois, pour prendre la seule valeur de ponctuant du discours (Caubet, 2001). Dans une configuration dissymétrique, qu'elle soit institutionnelle ou interpersonnelle, où s'établit un jeu de forces symboliques, l'insulte a un rôle perlocutoire spécifique. Contrairement aux fonctionnements entre pairs, l'insulte arrive généralement en clôture, dans une forme ultime de la montée en tension; c'est elle souvent, comme nous l'avons observé à travers les rapports d'enseignants (rapports qui relatent les conflits et justifient les punitions prises), qui déclenche la décision de sanction. Elle vient même après toute autre forme d'acte de langage négatif, comme le harcèlement ou la menace. À travers les corpus d'interactions en salle de classe nous avons pu montrer que l'insulte rend compte d'une transgression affirmée du rapport institutionnel et d'une remise en question de la légitimité de l'enseignant. Elle est parfois formulée à voix basse, voire cryptée en arabe, pour blesser devant témoin l'injurié (corpus Moïse, Auger, 2001), tandis que sa réalité ne peut être prouvée. On voit alors comment l'insulte surgit dans une montée en tension et vient, en dernier recours après d'autres procédés discursifs nombreux comme le dénigrement ou la menace. L'insulte, en particulier, use de sa forme essentialisante de façon radicale et réductrice dans les échanges en milieu informel, mais survient toujours après une certaine montée en tension. L'espace de la rue, a contrario, est caractéristique de la fulgurance de l'insulte (Moïse, à paraître). Il s'agit dans de telles situations soit d'exprimer une forme de tension (peur, par exemple), soit d'affirmer sa prise de pouvoir et de contrôle sur l'autre dans un effet de négation radicale. C'est ce que l'on observe par exemple dans les altercations entre automobilistes.

\section{Conclusion}

Ainsi, dans le cadre d'une sociolinguistique qui s'attache à décrire, par les pratiques langagières, les processus et les changements sociaux en œuvre, nous concevons une méthodologie globale et intégrative. De cette façon, selon les contextes d'énonciation et les enjeux interactionnels, l'usage d'outils d'analyse adaptés et diversifiés nous permet d'aborder et de décrire la complexité d'un phénomène social nommé comme « violence verbale ». Cette recherche nous pousse alors sans cesse à questionner la complexité du réel et la pertinence de nos méthodologies, mais aussi la place sociale et politique du chercheur. Nous ne pouvons faire l'impasse de la responsabilité que nous avons dans nos interventions de formation et dans la construction des discours sociaux. Les scientifiques détiennent un vrai pouvoir symbolique. Il ne faut pas croire que, pétris de bonnes intentions, nous allons échapper aujourd'hui à l'imprégnation au sein des discours de nos construits idéologiques ; nous participons aux représentations en marche, de la diversité culturelle et linguistique à «l'intégration» en France ou ici-même à la "citoyenneté ». Nos recherches peuvent servir de légitimation (ou a contrario de repoussoir, donc de contre-légitimation ce qui va, d'une 
certaine façon, dans le même sens) du politique. Il suffit sans doute de bien le savoir pour examiner avec auto-réflexion les conséquences de l'action de la recherche (Heller, M., 2000 :15).

\section{Bibliographie}

Auger, N., 2003, «Du dialogisme pour nommer l'autre en contexte scolaire interculturel», Actes du colloque Dialogisme et Nomination, Presses de l'Université Montpellier III, Praxiling, Jeunes chercheurs, 7-8 mars 2003 : $241-252$

Auger, N., 2005, , « Des malentendus constructifs en didactique des langues-cultures », Bacha, G., Laroux, G. et Séoud, A. (Eds), actes du colloque Le malentendu, du 15 au 17 avril 2004, Presses Internationales de la Faculté des Lettres de Sousse (Tunisie), éditions officielles de la République tunisienne : 285-292

Auger, N., Fracchiolla, B. et Moïse, C. 2005, «Réactions au texte de Michelle Van Hooland » M. Van Hooland, M. (Ed.) Psychosociolinguistique, Les facteurs psychologiques dans les interactions verbales, Paris, L'Harmattan : 95107.

Auger, N. et Moïse C., 2005, «Violence verbale : malentendu ou mésentente », Bacha, G., Laroux, G. et Séoud, A. (Eds), actes du colloque Le malentendu, du 15 au 17 avril 2004, Presses Internationales de la Faculté des Lettres de Sousse (Tunisie), éditions officielles de la République tunisienne : 293-302.

Auger N., et Moïse, C., 2006, « Pourquoi une analyse qualitative du discours et des interactions ? », Dugneanu, P., (Ed.) Analele Universitatii Ovidius, Serie Filologie, Tome XVI, 2005, Ovidius University Press, Roumanie : 171181.

Auger, N., 2007, «La relation médias/société en question : un cas de violence dans une banlieue de Perpignan », À l'école de la sécurité, Ville-Ecole-Intégration (VEI), n¹46, décembre : 45-53.

Austin, J., 1962, How to do things with words, ed. J. O. Urmson and Marina Sbisá. Cambridge , Mass., Harvard University Press.

Authier-Revuz, J., 1992, « Repères dans le champ du discours rapporté », L’information grammaticale, 55 : 38-42

Bakthine M., 1997, Le Marxisme et la philosophie du langage, Paris, Minuit.

Blanchet, P., 2000, Linguistique de terrain, méthode et théorie, une approche ethno-sociolinguistique, Presses Universitaires de Rennes.

Bourdieu, P, 1982, Ce que parler veut dire, Paris, Fayard.

Brown P., Levinson, S., 1978, «Universals in language use: Politeness phenomena »,Goody, E. (Ed). Questions and politeness. Strategies in social interaction, Cambridge, CUP : 56-289.

Brown P., Levinson, S., 1987, Politeness. Some universals in language use, CUP, Cambridge.

Budach, G., Moïse, C., Duchêne, A. et M. Richards, 2007, « Le bison, la fleur de lys et la feuille d'érable : vrais ou faux stéréotypes canadiens? », in Boyer, H. (Ed.), Stéréotypage, stéréotypes fonctionnement ordinaire et mise en scène, Paris, L'Harmattan : 29-45.

Caubet, D., 2001, « Du baba (papa) à la mère, des emplois parallèles en arabe marocain et dans les parlures jeunes en France », Cahiers d'études africaines (Langues déliées), 163-164 : 735-748.

Chambers, J., 1994, Sociolinguistic Theory, Oxford, Blackwell.

Fairclough, N, 1992, 1992, Discourse and Social Change, Cambridge, Polity Press.

Fracchiolla, B., (à paraître en mars 2008), «L'attaque courtoise: de l'usage de la politesse comme stratégie d'agression dans le débat Sarkozy-Royal du 02 mai 2007 », Actes des 9èmes Journées d'Analyse des Données Textuelles, Lyon, 12-14 mars 2008.

Fracchiolla, B., 2003, Ecologie et altérité : du discours de valeurs au discours de droits, thèse pour le doctorat, dir. M. Abdallah-Pretceille, université de Paris 3.

Fracchiolla, B, et Moïse, C., (à paraître) « Construction de la violence verbale et circulation des discours : autour du contrat première embauche », colloque Ci-dit, Université de Laval, Québec, Circulation des discours et liens sociaux : le discours rapporté comme pratique sociale, 7-9 octobre 2006, Montréal, Nota Bene. 
Gadet, F., 2000, «Vers une sociolinguistique des locuteurs », Le futur de la sociolinguistique européenne, Sociolinguistica, numéro $14: 99-103$

Galatalo, R. et R. Mizzau, 1998, «Conflit conversationnel et malentendu : quelques relations possibles », La linguistique 34-1 : 151-164

Goffman E., 1971/73, La mise en scène de la vie quotidienne, t1 La présentation de soi, t2 Les relations en public, Paris, Minuit.

Guilhaumou, J., 2005, «Où va l'analyse de discours? Autour de la notion de formation discursive », Maingueneau, D. (Ed.), Analyse du discours. Etat de l'art et perspective, Marges Linguistiques, revue électronique, $9: 95-113$.

Gumperz, J., 1982, Discourse Stratégies. Cambridge University Press. Trad. fçaise : Introduction à la sociolinguistique interactionnnelle, Paris, L'Harmattan, 1989.

Halliday, M., 1978, Language as social semiotics: The social interpretation of language and meaning, London, Edward Arnold.

Heller, M., 1995 « Le concept de "situation ”, les pratiques langagières et les rapports interethniques », in Véronique, D. et Vion, R. (Eds.), Des savoir-faire communicationnels, Aix-en-Provence, Presses de l'université de Provence : $87-102$.

Heller, M., 2002, Eléments d'une sociolinguistique critique, Paris, Didier.

Heller, M. et N. Labrie (Eds.), 2004, Discours et identités. Le Canada français, entre modernité et mondialisation, chapitre 1, Fernelmont, Belgique, Editions modulaires européennes.

Héritier, F., 1999, « Les matrices de l'intolérance et de la violence », Héritier, F (Ed.), De la violence, Paris, Editions Odile Jacob, Opus : 321-344

Huston, H., 2002, Dire et interdire, Paris, Payot.

Hymes, D., 1974, Foundations in Sociolinguistics. An Ethnographic Approach, Philadelphia, University of Pennsylvania Press.

Kerbrat-Orecchioni C., 1992, Les interactions verbales t2, Paris, Armand Colin.

Kerbrat-Orecchioni, C., 1997, L'énonciation, Paris, Armand Colin.

Kress G. et T. van Leeuwen, 1996, Reading Images - The Grammar of Visual Design, London, Routledge.

Lagorgette, D. et Larrivée, P., 2004, «Interprétation des insultes et relations de solidarité », Langue française, numéro $144: 83-104$.

Mesthrie, R. (Ed.), 2001, Concise Encyclopedia of Sociolinguistics, Amsterdam.

Moïse, C. 2002, «Pour quelle sociolinguistique urbaine ?», in Pratiques langagières urbaines, enjeux identitaires, enjeux cognitifs, VEI Enjeux, numéro 130, Centre de documentation pédagogique, Paris : 75-87.

Moïse, C. 2003, «Des configurations urbaines à la circulation des langues... ou... les langues peuvent-elles dire la ville? », Frontières et territoires urbains, les frontières sociolinguistiques, Journée internationale de sociolinguistique urbaine, Kénitra, Maroc, 12 décembre 2003, in Thierry Bulot, T. et Messaoudi, L. (dir.), Sociolinguistique urbaine (frontières et territoires), Éditions Modulaires Européennes, Cortil-Wodon, Belgique : $53-80$

Moïse, C., 2007a), «Déambulations sociolinguistiques », Lambert P., Millet A., Rispail M. et C.Trimaille (Eds.), Variations au cœur et aux marges de la sociolinguistique. Mélanges offerts à Jacqueline Billiez, L'Harmattan, Paris : 179-186.

Moïse, C. 2007b), «Contexte et violence verbale », La mise en œuvre des langues dans l'interacton, 4ème colloque du réseau de sociolinguistique, Paris, 7-8 octobre 2005, collection Espaces discursifs, L’Harmattan : 79-101

Moïse, C., (À paraître) «Espace public et fonction de l'insulte dans la violence verbale », Les insultes en français : de la recherche fondamentale à ses applications, colloque de Chambéry, 30 mars-1er avril 2006

Mondada, L., 1998, «De l'analyse des représentations à l'analyse des activités descriptives en contexte », Linguistique et représentations, Cahiers de praxématique, Université Paul Valéry, n³1, Montpellier III : 115148. 
Mougin, S. (Ed), 2006, La médisance, Presses universitaires de Reims.

Ott, H., 1997, « L’approche constructive des conflits », Cahiers de la Réconciliation, n 1-2.

Pêcheux, M., 1975, Les vérités de La Palice. Linguistique, sémantique, philosophie, Paris, Maspero.

Romain, C., 2006, «Evaluation de l'impact des stéréotypes en milieu scolaire hétérogène », Actes du Colloque international de Montpellier III - 21, 22 et 23 juin 2006 , Boyer, H. (Ed)., Stéréotypage, stéréotypes : fonctionnements ordinaires et mises en scène, Tome 3, L'Harmattan, Paris : 203-212.

Romain, C., 2006, «Evaluation de l'impact des stéréotypes en milieu scolaire hétérogène », Actes du Colloque international de Montpellier III - 21, 22 et 23 juin 2006 , Boyer, H. (Ed)., Stéréotypage, stéréotypes : fonctionnements ordinaires et mises en scène, Tome 3, L'Harmattan, Paris : 203-212.

Romain, C., 2007, «Faits prosodiques et faits discursifs dans la construction de la relation interpersonnelle enseignant / élève(s) au collège ", Pré-Actes de la deuxième édition du Symposium International sur les interfaces discours / prosodie, Université de Genève, Suisse - 12, 13 et 14 septembre 2007, Nouveaux Cahiers de linguistique française, $\mathrm{n}^{\circ}$ 28, Genève, Suisse : 137-145.

Romain, C., 2008, «Description de la violence verbale en situation difficile d'enseignement », Moïse, C., Auger, N., Fracchiolla, B. et Schultz-Romain, C. (Eds De l'impolitesse à la violence verbale, volume 2, collection Espaces discursifs, Thierry Bulot, L'Harmattan : 97-120.

Rosier, L. 1999, Le discours rapporté. Histoire, théories, pratiques, Louvain-la-Neuve, Duculot.

Rosier, L., 2005, «L'analyse du discours et ses corpus. À travers le prisme du discours rapporté », in Maingueneau, D. (éd.), Analyse du discours. Etat de l'art et perspective, Marges Linguistiques, revue électronique, 9 : 154-165.

Searle, J., (1972), Les actes de langage, Paris, Hermann.

Traverso, V., 1996, La conversation familière, Presses universitaires de Lyon : 184-193

Van Hooland, M., 2002, La parole émergente. Approche psychosociolinguistique de la résilience. Parcours théoricobiographique, L'Harmattan.

Vincent, D., 2005, «La qualification péjorative dans tous ses états », Langue française, 144 : 59-81.

Vion R., 1999, «Linguistique et communication verbale », Gilly, M., Roux, J.-P. et A. Trognon (Eds.), Apprendre dans l'interaction. Analyse des médiations sémiotiques, Presses Universitaires de Nancy et de l'Université de Provence : 41-67.

Watzlawick, P., J. Weakland, J et R. Fisch, 1975, Changements, Points Seuil.

Williams, G., 1992, Sociolinguistics. A sociological Critique, London, Routledge.

\footnotetext{
${ }^{1}$ Ce texte est une synthèse des approches et réflexions que nous menons depuis plusieurs années. Le projet de recherche, La violence verbale, a été financé par La Délégation Interministérielle à la Ville et la Région Provence Alpes Côte d'Azur. Il reçoit également le soutien depuis janvier 2008 de la MSH Paris Nord et de la MSH de Montpellier.

${ }^{2}$ Un site qui rendra compte à la fois de notre DVD, de nos publications, séminaires, et formations est en préparation. Il sera hébergé par l'université d'Avignon.

3 Le logiciel Lexico3 est développé par le SYLED à l'université Paris 3 - Sorbonne nouvelle. Pour plus d'information: http://www.cavi.univ-paris3.fr/ilpga/ilpga/tal/lexicoWWW/.

${ }^{4} \mathrm{C}$ 'est pourquoi, également, l'analyse statistique qui permet de rechercher les lexèmes spécifiques s'avère parfois un précieux outil.
} 